Journal of Computer Science 7 (2): 173-178, 2011

ISSN 1549-3636

(C) 2011 Science Publications

\title{
A Novel Approach to Head positioning using Fixed Center Interpolation Net with Weight Elimination Algorithm
}

\author{
Mahmoud Zaki Iskandarani \\ Faculty of Science and Information Technology, Department of Computer Science, \\ Al-Zaytoonah University of Jordan, \\ P.O. Box 911597, Post Code 11191, Amman, Jordan
}

\begin{abstract}
A reliable algorithm for head movements inside a vehicle is designed. The proposed algorithm allowed the adjustment of basic functions such as indicators, mirrors and reverse lights based on the driver final head position. The algorithm system mapped a predefined coordinates for driver's head that resulted in a computable geometry via a sensory system which is fed to the vehicle actuating system. Problem statement: Head position recognition is one of the most common problems encountered in engineering and scientific disciplines, which involves developing prediction or classification models from historic data or training samples. In the past few years face detection and person identification became important issues due to security concerns, leading to head gesture algorithm development and implementation. Approach: This study introduces a new approach that combines Fixed Center Interpolation Net Algorithm (FCIN) with Wight Elimination Algorithm (WEA). This enhances the ability to classify and predict head positions and poses and gives better representation capabilities for the overall system algorithm. Such algorithm is able to handle pattern recognition problems using Radial Basis Function (RBF) models. The system algorithm has been developed based on the mathematical properties of the interpolation and design matrices of RBF models. Results: A reliable, fast and robust approach for driver head position recognition is achieved and presented. Conclusion: A simple hybrid algorithm for driver's head movements is designed and tested. The obtained results proved the algorithm applicability and ability to predict and act upon head gestures.
\end{abstract}

Key words: Fixed Center Interpolation Net (FCIN), Weight Elimination Algorithm (WEA), automotive collisions, head gesture, Radial Basis Function (RBF), enable driver-assisted systems, input vector, neural network, neural fuzzy systems

\section{INTRODUCTION}

Driver distraction is a prominent cause of automotive collisions. To enable driver-assisted systems to address these problems, we need new algorithm to infer a driver's focus of attention.

Vehicle and driver safety relies on the ability of the driver to be focused on driving rather than using various gadgets to enforce actions. As new vehicles and obstacles move into the vicinity of the car, a driver must be aware of the change and be ready to respond as necessary. When a driver fails to initiate an action, there is an increased potential for a life-threatening collision (Doshi et al., 2009; Junker et al., 2008; Murphy-Chutorian and Trivedi, 2009; 2010).

Gesture recognition is a complex task which involves many aspects such as motion modelling, motion analysis, pattern recognition, machine learning and neural fuzzy systems. Gestures are expressive and meaningful body motions used in daily life as means of communication where a computer based automatic recognition system is necessary for interpretation and signal control in an interactive and dynamic environment (Roomi et al., 2010; Srinivasa and Grossberg, 2008; Suk et al., 2010; Iskandarani, 2010; $\mathrm{Wu}$ and Trivedi, 2008).

. In such an environment body motion can be defined as a sequence of states in a configurable space, which can be modeled, based on the following principles:

- Static start and end position

- Smooth transition forward and backward

There is a need to have on-board systems in vehicles with capability to operate in harsh environments. Such systems are designed to gather and process information in order to carry out the actions necessary to achieve their designated functions. Such 
systems operate using reaction models. The Brain of the system reacts to impulses, picked up by the sensors and transmits information and orders to the actuators, of the system. All of the components, materials and software for these systems satisfy requirements concerning size, sturdiness, energy consumption and immunity to external disturbances; the key words are: security, reliability, quality, safety, real-time, autonomy and servo assistance.

In this study a novel procedure for static head-pose estimation and a new algorithm for head gesture position are presented. Visual tracking is integrated into the novel FCIN system for measuring the position and orientation of a head (Asamwar et al., 2010; Toure and Beiji, 2010; Bohme et al., 2008; Chan et al., 2010; Attarzadeh and Ow, 2010).

. This system consists of interconnected modules that detect head position, provide initial estimates of the head's pose and can be implemented to continuously track head position and orientation.

\section{MATERIALS AND METHODS}

Figure 1 show the grid used to map head movements. The grid is scanned in one-dimension multiple times starting from a certain location, then returning back to the location below the starting point $(y+\Delta y)$. The used number of nodes will obviously affect accuracy and speed of convergence of the developed algorithm.

Figure 2 show a photo used to test the proposed system with Fig. 3-5 demonstrate three different positions namely; Right, Left and Center, while Fig. 6-8 show the photo at different angles namely; $30^{\circ}, 45^{\circ}$ and $90^{\circ}$.

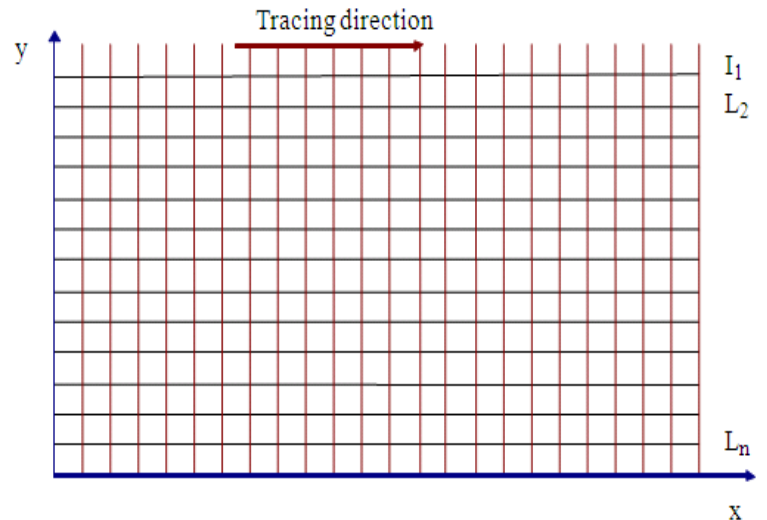

Fig. 1: System sampling gird

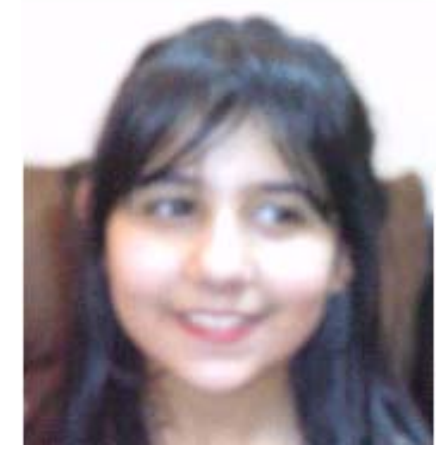

Fig. 2: Adla Iskandarani's Photo used to test the system

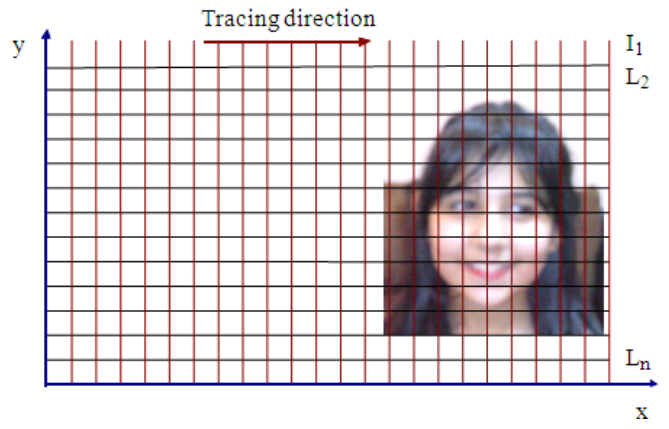

Fig. 3: Far right

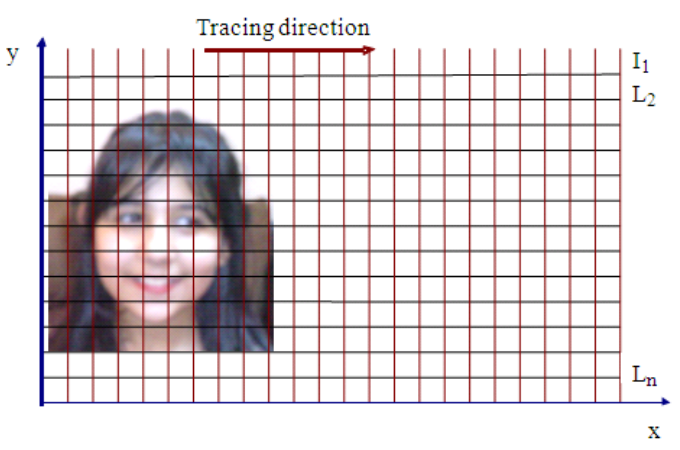

Fig. 4: Far left

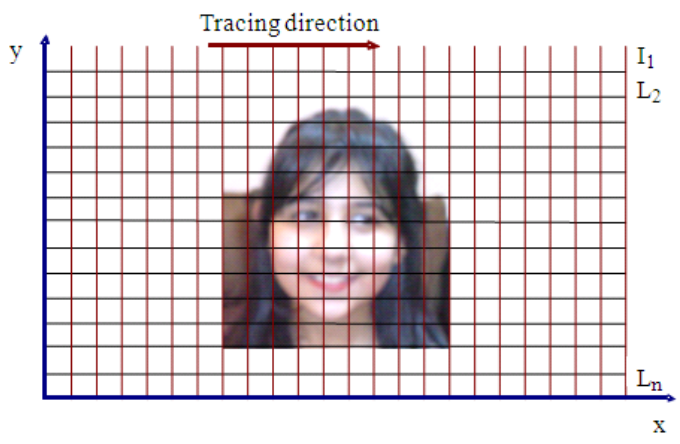

Fig. 5: Center (reference sampled image). 


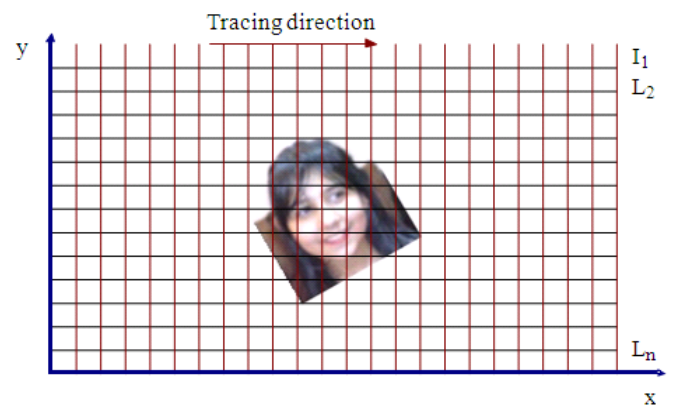

Fig. 6: $30^{\circ}$ angle

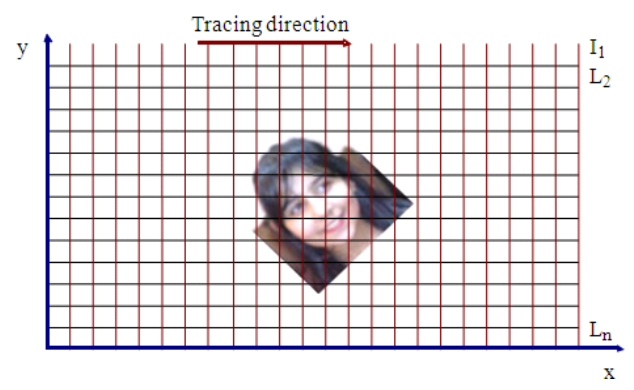

Fig. 7: $45^{\circ}$ angle

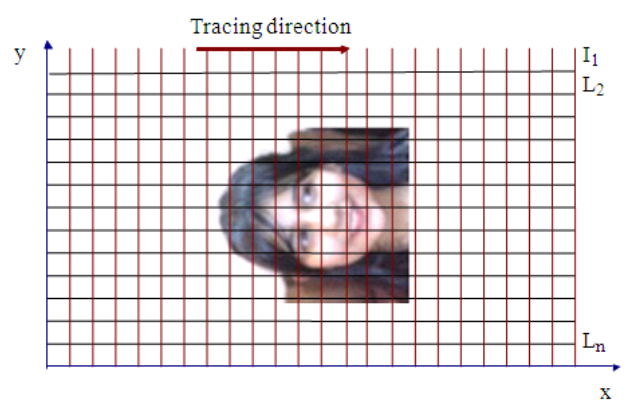

Fig. 8: $90^{\circ}$ angle

For a unidirectional, two-dimensional (two-layer) interpolation Gaussian network with the ability to predict an output $\mathrm{y}$ for a sample of inputs $\left(\mathrm{x}_{1}, \ldots, \mathrm{x}_{\mathrm{n}}\right)$, the output is related to the inputs via a weighted shaping function given by:

$$
y\left(x_{1}, \ldots, x_{n}\right)=\sum_{i=1}^{s} w_{i} f\left(x_{1}, \ldots, x_{n}\right)
$$

The function in (1) needs to operate under the conditions:

- The output values should be exactly equal to the values of the training set if training inputs are used

- The output values should be close to the outputs of the training set if other inputs are used
- $\quad f$ should reach a maximum or minimum peak value when the input values $\left(\mathrm{x}_{1}, \ldots, \mathrm{x}_{\mathrm{n}}\right)$ are close to the centre vector

For $\left(\mathrm{x}_{1}, \ldots, \mathrm{x}_{\mathrm{n}}\right)$ fulfilling the following conditions:

- Considered as coordinates of vector $\mathrm{x}$

- Associated with the centre vector R

Then:

$\mathrm{f}\left(\mathrm{x}_{\mathrm{i}}\right)=\mathrm{h}\left(\left\|\mathrm{x}_{\mathrm{i}}-\mathrm{R}\right\|\right)$

This means that each is handling the influence of the reference vector and the input vector.

The Gaussian representation for (2) is given by:

$$
\begin{aligned}
& \mathrm{f}\left(\mathrm{x}_{\mathrm{i}}\right)=\mathrm{h}\left(\left\|\mathrm{x}_{\mathrm{i}}-\mathrm{R}\right\|\right)=\mathrm{e}^{-\frac{1}{2 \sigma} \mid \mathrm{x}_{\mathrm{i}}-\mathrm{R} \|^{2}} \\
& \text { From (1): } \\
& \mathrm{y}\left(\mathrm{x}_{\mathrm{i}}\right)=\mathrm{w}_{\mathrm{i}} \mathrm{e}^{-\frac{1}{2 \sigma_{\mathrm{I}}}\left\|\mathrm{R}-\mathrm{x}_{\mathrm{i}}\right\|^{2}}
\end{aligned}
$$

Where, $\sigma$ controls generalization and function spread, with transition from local (low $\sigma$ values) to global (high $\sigma$ values) and $\mathrm{w}_{\mathrm{i}}$ represents the associated set of weights.

For head positions using MGIN algorithm, the weight matrix is a function of position and orientation and is given by Eq. 5:

$\mathrm{W}_{\mathrm{i}}=\mathrm{e}^{-\frac{1}{2 \sigma_{2}}\left\|1-\cos \left(\theta_{\mathrm{i}}\right)\right\|^{2}}$

where, $\theta$ is the angle between the new head position sampled image and the reference sampled one.

The centre vector $\mathrm{R}$ represents a reference ratio of existing image to the grid image, without the presence of a driver. Hence it is computed as having a fixed value of 1 .

The input vector values $\mathrm{x}$ are computed as:

$\mathrm{x}=\frac{\mathrm{M}}{\mathrm{G}}$

Where, $\mathrm{M}$ is the mixed grid-driver image with the condition: $\mathrm{M} \rightarrow \mathrm{G}$ (No one in the vehicle)

So for each value of scanned line in the x-direction per y-value, (4) becomes:

$$
\mathrm{g}\left(\mathrm{x}_{\mathrm{i}}\right)=\mathrm{e}^{-\frac{1}{2 \sigma_{2}} \|-\left.\cos \theta\right|^{2}} \mathrm{e}^{-\frac{1}{2 \sigma_{\mathrm{I}}} \mid \frac{\mathrm{M}}{\mathrm{G}}-\mathrm{x}_{\mathrm{i}} \|^{2}}
$$




\section{RESULTS}

Figures 2-8 shows simulated images of Adla Iskandarani used to test the mathematical model and associated algorithms. Figure 9 shows the WEA based neural network structure used for prediction of angles based on driver's head movements, while Table 1 holds FCIN figures used to train the network with Table 2 showing the predicted FCIN figures of $\mathrm{g}(\mathrm{x})$. The effect of $\sigma$ on accuracy and generalization of $\mathrm{g}(\mathrm{x})$ is illustrated in Fig. 10-12 with angle function mapping of image sampling shown in Fig. 13.

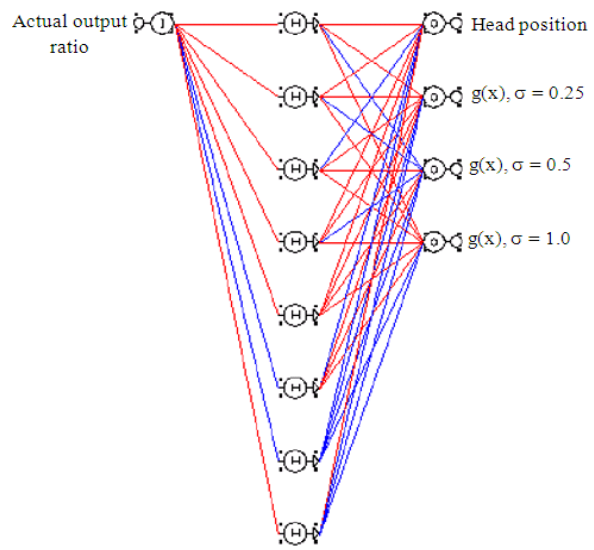

Fig. 9: Training network

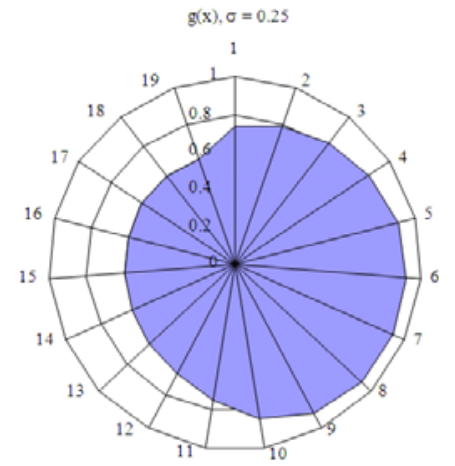

Fig. 10: Interpolation for $\sigma=0.25$

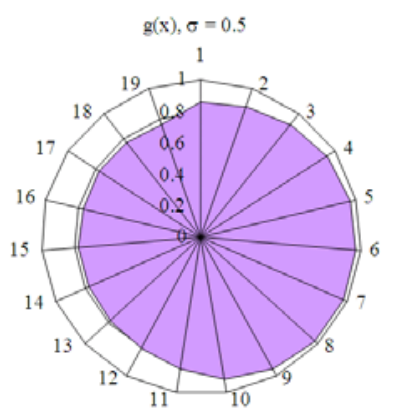

Fig. 11: Interpolation for $\sigma=0.5$
Table 1: Training set

\begin{tabular}{lllll}
\hline & & $\mathrm{g}(\mathrm{x})$ & & \\
& & & \\
& $\begin{array}{l}\text { Actual output } \\
\text { ratio }\end{array}$ & $\sigma=0.25$ & $\sigma=0.5$ & $\sigma=1.0$ \\
\hline No driver & 1.00 & 1.000 & 1.000 & 1.000 \\
Shifted right & 1.30 & 0.835 & 0.914 & 0.960 \\
Shifted left & 1.35 & 0.795 & 0.891 & 0.944 \\
Centre & 1.40 & 0.738 & 0.860 & 0.927 \\
$30^{\circ}$ & 0.87 & 0.933 & 0.980 & 0.990 \\
$45^{\circ}$ & 0.94 & 0.840 & 0.913 & 0.960 \\
$90^{\circ}$ & 1.50 & 0.595 & 0.771 & 0.878 \\
\hline
\end{tabular}

Table 2: Prediction set

\begin{tabular}{lllll}
\hline & & Predicted $g(\mathrm{x})$ & & \\
Actual & Head & & & \\
output ratio & position $\left(\theta^{\circ}\right)$ & $\sigma=0.25$ & $\sigma=0.5$ & $\sigma=1.0$ \\
\hline 1 & No driver & 1.000000 & 1.000000 & 1.000000 \\
1.400001 & 0 & 0.738000 & 0.860000 & 0.927000 \\
1.341926 & 5 & 0.775968 & 0.888024 & 0.939228 \\
1.246023 & 10 & 0.822079 & 0.918466 & 0.954067 \\
1.115254 & 15 & 0.868327 & 0.945982 & 0.968972 \\
0.988497 & 20 & 0.904290 & 0.965750 & 0.980617 \\
0.906664 & 25 & 0.925327 & 0.976673 & 0.987475 \\
0.86999 & 30 & 0.932996 & 0.980002 & 0.989999 \\
0.862696 & 35 & 0.927944 & 0.975406 & 0.988377 \\
0.881077 & 40 & 0.902695 & 0.956902 & 0.980221 \\
0.94001 & 45 & 0.840003 & 0.912999 & 0.960001 \\
1.057947 & 50 & 0.744615 & 0.851336 & 0.928953 \\
1.206685 & 55 & 0.666027 & 0.805510 & 0.902826 \\
1.326089 & 60 & 0.624435 & 0.783156 & 0.888683 \\
1.399459 & 65 & 0.605739 & 0.773683 & 0.882205 \\
1.441357 & 70 & 0.597642 & 0.769903 & 0.879333 \\
1.466068 & 75 & 0.594414 & 0.768737 & 0.878127 \\
1.481691 & 80 & 0.593575 & 0.768889 & 0.877735 \\
1.492314 & 85 & 0.593968 & 0.769753 & 0.877760 \\
1.499995 & 90 & 0.594998 & 0.771001 & 0.877999 \\
\hline
\end{tabular}

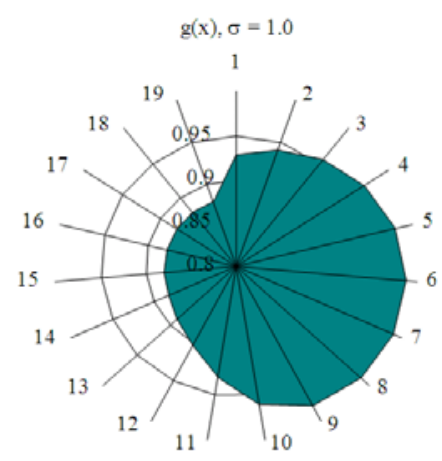

Fig. 12: Interpolation for $\sigma=1.00$

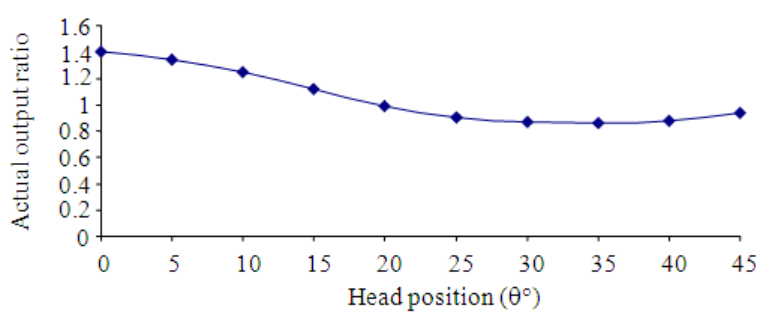

Fig. 13: Relationship between head position and classification function output 


\section{DISCUSSION}

The used combined FCIN and WEA algorithms made use of the classification property of the developed modified interpolation net function and the weight elimination property of the WEA algorithm. This enabled a fast and more flexible yet accurate simulation of head poses to be achieved with minimum memory requirements (Choi and Kim, 2008; Dornaika and Davoine, 2008; Jenab and Rashidi, 2009; Yogameena et al., 2010; Harkouss et al., 2010).

From Table 1 and 2 and Fig. 10-13, the following is realized:

- The effect of adding the extra term and modifying the original RBF function, which resulted in mapping of head poses

- The correlation between the input to the actuators and the diver head position detected by the sensing system is achieved

- Difference in angled position of driver's head is related to accumulative pixel concentration per coordinate which is mapped by the sensing and processing algorithms

- The ability to construct a full spectrum of sequences with each sequence representing a type of image resulted from a different head position

- The used FCIN algorithm is based on the Energy Band Model, which assumes that image pixel values and distribution in energy levels is modified and modulated

\section{CONCLUSION}

The combined FCIN-WEA algorithms unifies through conversion the extracted information from irrelevant background and correlate obtained data to initiate an action based on a driver head position. Also, the known difficulty in the interpretation of input data is resolved through the algorithm. This very successful approach to head pose classification is further supported by its ability to correlate various input technologies.

\section{REFERENCES}

Asamwar, R.S., K. Bhurchandi and A.S. Gandhi, 2010. Successive image interpolation using lifting scheme approach. J. Comput. Sci., 6: 969-978. DOI: $10.3844 /$ jcssp. 2010.969 .978

Attarzadeh, I. and S.H. Ow, 2010. A novel algorithmic cost estimation model based on soft computing technique. J. Comput. Sci., 6: 117-125. DOI: 10.3844/jcssp.2010.117.125
Bohme, M., M. Haker, T. Martinetz and E. Barth, 2008. A facial feature tracker for human-computer interaction based on 3D Time-Of-Flight cameras. Int. J. Int. Syst. Technol. Appl., 5: 264-273: DOI: 10.1504/IJISTA.2008.021289

Chan, L.H., S.H. Salleh and C.M. Ting, 2010. Face biometrics based on principal component analysis and linear discriminant analysis. J. Comput. Sci., 6: 693-699. DOI: $10.3844 /$ jcssp.2010.693.699

Choi, S. and D. Kim, 2008. Robust head tracking using 3D ellipsoidal head model in particle filter. Patt. Recognit., 41: 2901-2915: DOI: 10.1016/j.patcog.2008.02.002

Dornaika, F. and F. Davoine, 2008. Simultaneous facial action tracking and expression recognition in the presence of head motion. Int. J. Comput. Vis., 76: 257-281. DOI: 10.1007/s11263-007-0059-7

Doshi, A., S.Y. Cheng and M.M. Trivedi, 2009. A novel active heads-up display for driver assistance. IEEE Trans. Syst. Man Cybernetics-Part B: Cybernetics, 39: 85-93. DOI: 10.1109/TSMCB.2008.923527

Harkouss, Y., S. Mcheik and R. Achkar, 2010. Accurate wavelet neural network for efficient controlling of an active magnetic bearing system. J. Comput. Sci., 6: 1457-1464. DOI: 10.3844/jcssp.2010.1452.1459

Iskandarani, M.Z., 2010. Head gesture analysis using matrix group displacement algorithm. J. Comput. Sci., $\quad 6$ : $1362-1365$. DOI: $10.3844 /$ jcssp. 2010.1362 .1365

Jenab, K. and K. Rashidi, 2009. Fuzzy bayesian condition monitoring model based on exponential distribution. Int. J. Eng. Technol., 1: 172-178.

Junker, H., O. Amft, P. Lukowicz and G. Troster, 2008. Gesture spotting with body-worn inertial sensors to detect user activities. Pattern Recognit., 41: 2010-2024. DOI: 10.1016/j.patcog.2007.11.016

Murphy-Chutorian, E. and M. Trivedi, 2009. Head pose estimation in computer vision: A survey. IEEE Trans. Pattern Anal. Mach. Intell., 31: 607-626. DOI: 10.1109/TPAMI.2008.106

Murphy-Chutorian, E. and M.M. Trivedi, 2010. Head pose estimation and augmented reality tracking: An integrated system and evaluation for monitoring driver awareness. IEEE Trans. Intel. Trans. Syst., 11: 300-311. DOI: 10.1109/TITS.2010.2044241

Roomi, S.M.M., R.J. Priya and H. Jayalakshmi, 2010. Hand gesture recognition for human-computer interaction. J. Comput. Sci., 6: 1002-1007. DOI: 10.3844/jcssp.2010.1002.1007 
Srinivasa, N. and S. Grossberg, 2008. A head-neck-eye system that learns fault-tolerant saccades to 3-D targets using a self-organizing neural model. Neural Networks, 21: 1380-1391. DOI: 10.1016/j.neunet.2008.07.007

Suk, H., B.K. Sin and S.W. Lee, 2010. Hand gesture recognition based on dynamic Bayesian network framework. Patt. Recog., 43: 3059-3072: DOI: 10.1016/j.patcog.2010.03.016

Toure, M.L. and Z. Beiji, 2010. Intelligent sensor for image control point of eigenfaces for face recognition. J. Comput. Sci., 6: 484-491. DOI: $10.3844 /$ jcssp.2010.484.491
$\mathrm{Wu}, \mathrm{J}$. and M. Trivedi, 2008. A two-stage head pose estimation framework and evaluation. Patt. Recog., 41: 1138-1158. DOI: 10.1016/j.patcog.2007.07.017

Yogameena, B., E. Komagal, M. Archana and S.R. Abhaikumar, 2010. Support vector machinebased human behavior classification in crowd through projection and star skeletonization. J. Comput. Sci., 6: 1008-1013. DOI: 10.3844/jcssp.2010.1008.1013 